\title{
The prescription opioid epidemic: a call to action for our profession
}

\author{
Norm Buckley, MD
}

Received: 9 October 2015/Accepted: 15 October 2015/Published online: 23 October 2015

(C) Canadian Anesthesiologists' Society 2015

There is no question that too many people die from the use and abuse of opioids. The article by Alam and Juurlink in this issue of the Journal provides an overview of the issue of the prescription opioid epidemic ${ }^{1}$ and contains several useful messages for anesthesiologists which I summarize in this editorial. In some way, however, the most important message for our profession is in the conclusion-i.e., anesthesiologists have a leadership role to play in the treatment of chronic pain. Understanding the limitations and value of opioids, as with all drugs, is crucial. There is an opportunity here for anesthesiologists to provide direction and to enhance knowledge, and there are several mechanisms through which to do so.

Problem: Far too many people die from inappropriate (and sometimes appropriate) use of opioids.

We don't always use opioids according to the best evidence available or screen patients for risk of opioid abuse and manage their condition appropriately. That being said, there are potential solutions. As a profession, we are leaders in the practice of safety with an enviable improvement in anesthesia outcomes over the past 50 years. We have accomplished this by understanding our business through conducting systematic research, enhancing technical and pharmacological capabilities, and to a large extent, increasing our personal vigilance during anesthetic care. The same principles should apply to our practice vis-à-vis prescription opioids. Although Alam and Juurlink dismiss a national opioid guideline ${ }^{2}$ as "often not followed", the necessary information to support rational and appropriate use of opioids is well described in that

N. Buckley, MD ( $ه)$

Department of Anesthesia, Michael G. DeGroote School of Medicine, McMaster University, 1280 Main St W, Hamilton, ON, Canada guideline. In fact, the Annals of Internal Medicine reported that the "Canadian Guideline for Safe and Effective Use of Opioids for Chronic Non-Cancer Pain" is one of two guidelines that are highly rated according to the AGREE system of rating guideline development and would be used "without modification" by more than $50 \%$ of the guideline reviewers. ${ }^{3}$ Using and teaching others to use this guideline could well lead to significant improvement in care.

There is a national strategy to address prescription drug abuse. It is not only opioids that are problematicstimulants and sedatives are also an issue-but in any event, the problem of opioid abuse is significant. The strategy entitled "First Do No Harm: Responding to Canada's Prescription Drug Crisis" was announced by the Canadian Centre on Substance Abuse in $2014 .{ }^{4}$ The strategy was developed and endorsed by over 30 organizations across the country, including the Canadian Pain Society, opinion leaders (such as Dr. Irfan Dhalla), public health, law enforcement, First Nations, dentistry, pharmacy, the Institute for Safe Medication Practices, patients, relatives of patients, victims of drug abuse or drug-related adverse events, the research-based pharmaceutical industry, and others. Out of this strategy came 55 specific initiatives on which to take action. Such initiatives include significantly enhancing the education of healthcare professionals on the topics of both pain and addiction and their management. A lack of knowledge and access to care in these areas has been identified as one of the root causes of widespread prescribing of opioids for pain in the absence of substantial evidence to support this practice.

Problem: Pain and addiction are two poorly understood problems for which optimal treatment is often not available

There are potential solutions to the above problem. We need more knowledge about pain assessment and 
management, greater awareness of the risks of addiction, and the expertise to identify dependence when it occurs. Moreover, to support these fundamentals, we need better access to optimal treatment. We should continue to be leaders in a field (i.e., pain management) where we have arguably led the way for a generation or more. The Royal College of Physicians and Surgeons of Canada has recently approved a Pain Medicine residency, and Western University will have the first Canadian graduate of this program within the year. This residency submission was supported by the Canadian Anesthesiologists' Society along with the Canadian Pain Society and the Association of Canadian University Departments of Anesthesia. The process was led by anesthesiologist Patricia Morley-Forster from Western University. The coming generation of pain specialists will be drawn from many specialities, and this is one mechanism through which the problem of inappropriate use of opioids can be addressed and supported by our profession. The Canadian College of Family Physicians is developing a certificate of added competency in Pain Management, a process that will unfold over the next few years and, once again, is an opportunity for our experts to provide leadership.

Whether or not you subscribe to the concept of "gateway drugs" or addiction arising from limited exposure (e.g., the perioperative environment), certainly a primary objective must be for us to pursue the most appropriate, successful, and safe practice in providing perioperative care and analgesia. We have a leadership role in both perioperative and pain medicine and need to be actively engaged with our colleagues in surgery, psychology, and rehabilitation science. We must ensure that we contribute to health, not harm, and that care is not siloed into brief encounters. We must be aware of and engaged in the entire arc of a patient's encounter with our system.

We haven't gained enough knowledge about the longterm impact of opioid use, and therefore, we must advocate for the pursuit of appropriate research. We need to propose studies and participate in these studies, and we need to create registries that facilitate follow-up strategies so we can ensure that what we do actually helps our patients.

Some may find the article by Alam and Juurlink to be off-putting or perhaps even irritating because of its source, its content, or its style. "Get over it" and look for the messages. Anesthesiologists must pay attention to certain key messages and take notice of national initiatives in which we should be involved. This is, in my view, the central message to take from the work. This paper represents a call to action-i.e., to participate in the dissemination of the best possible care, to direct research that informs that care, and to educate coming generations of healthcare professionals in the skills and knowledge necessary to promote health and avoid the creation of iatrogenic healthcare issues.

\section{L'épidémie d'opioïdes d'ordonnance: il est temps pour notre profession d'agir!}

Il ne fait aucun doute qu'il y a trop de décès liés à l'utilisation et à l'abus d'opioïdes. L'article d'Alam et Juurlink, publié dans ce numéro du Journal, donne une vue d'ensemble du problème de l'épidémie d'opioïdes d'ordonnance 1 et comporte plusieurs messages utiles que je résumerai dans cet éditorial. D'une certaine manière cependant, le message le plus important à retenir pour notre profession est transmis dans leur conclusion : les anesthésiologistes doivent prendre le leadership dans le traitement de la douleur chronique. Pour ce faire, il est essentiel de bien comprendre les limites et l'utilité des opioïdes - tout comme c'est le cas pour n'importe quel médicament. Nous avons la possibilité, en tant qu'anesthésiologistes, de guider les connaissances dans ce domaine et de les améliorer, et il existe de nombreuses façons d'y parvenir.

Le problème : beaucoup trop de gens meurent d'une utilisation inadaptée (et parfois adaptée) des opiö̈des.

Nous n'utilisons pas toujours les opiö̈des selon les meilleures données probantes publiées, ni ne dépistons les patients à risque d'abuser des opioïdes afin de les prendre en charge de façon adéquate. Ceci étant dit, il existe des solutions potentielles. Notre profession est à la pointe en matière de pratiques sécuritaires, et nous pouvons nous targuer d'améliorations enviables en matières de pronostics liés à l'anesthésie depuis 50 ans. Nous avons réussi cet exploit en comprenant mieux notre métier, notamment en procédant à des recherches méthodiques, en améliorant nos capacités techniques et pharmacologiques et, dans une large mesure, en faisant preuve d'une vigilance accrue pendant la fourniture des soins anesthésiques. Ces mêmes principes devraient s'appliquer aux opioïdes d'ordonnance. Bien qu'Alam et Juurlink fassent peu de cas des lignes directrices nationales concernant les opioïdes ${ }^{2}$ car elles sont « souvent non respectées », les informations dont nous avons besoin pour utiliser les opioïdes de façon raisonnée et adaptée sont décrites avec précision dans ce document. En fait, selon la revue Annals of Internal Medicine, les Lignes directrices canadiennes sur l'utilisation sécuritaire et efficace des opiö̈des pour la douleur chronique non cancéreuse sont l'un de deux documents très bien cotés d'après le système AGREE d'évaluation de l'élaboration 
de lignes directrices, et elles pourraient être utilisées « sans modification » par plus de $50 \%$ des réviseurs des lignes directrices. ${ }^{3}$ L'utilisation de ces lignes directrices, et le fait d'enseigner à d'autres comment les utiliser-voilà l'une des façons d'améliorer considérablement les soins que nous prodiguons à nos patients.

Il existe une stratégie nationale visant à régler le problème d'abus des médicaments d'ordonnance. Les opioïdes ne sont pas les seuls à poser problème (les stimulants et les sédatifs s'ajoutent à cette liste), mais le problème d'abus des opioïdes est colossal, quoi qu'on en dise. La stratégie S'abstenir de faire du mal : Répondre à la crise liée aux médicaments d'ordonnance au Canada a été annoncée en 2014 par le Centre canadien de lutte contre les toxicomanies. ${ }^{4}$ Elle a été mise au point et endossée par plus de 30 organismes partout au pays-la Société canadienne de la douleur, des leaders d'opinion (tels que Dr Irfan Dhalla), les organismes de santé publique, les forces de l'ordre, les Premières nations, la médecine dentaire, la pharmacie, l'Institut pour l'utilisation sécuritaire des médicaments, des patients, des familles de patients, des victimes d'abus de médicaments ou de complications liées aux médicaments, l'industrie pharmaceutique fondée sur la recherche, et d'autres encore. De cette stratégie sont issues 55 initiatives spécifiques et concrètes. Parmi ces initiatives, citons le besoin d'améliorer drastiquement la formation des professionnels de la santé en matière de douleur et de toxicomanie, et de leur procurer des outils pour la prise en charge de ces conditions. L'une des principales causes identifiées de prescription répandue d'opioïdes pour le traitement de la douleur est le manque de connaissances et d'accès aux soins dans ces domaines et ce, malgré l'absence de données probantes suffisantes pour justifier une telle pratique.

Le problème : la douleur et la toxicomanie sont deux problèmes mal compris et pour lesquels un traitement optimal n'est souvent pas disponible.

Il existe des solutions potentielles à ce problème. Il faut commencer par approfondir nos connaissances en matière d'évaluation et de prise en charge de la douleur. Nous devons être conscients des risques de toxicomanie et pouvoir l'identifier lorsqu'elle survient. Et, dans les deux cas, nous avons besoin d'un meilleur accès à un traitement optimal. Nous devrions continuer d'être les chefs de file dans le domaine de la prise en charge de la douleur, domaine duquel nous sommes probablement à la tête depuis plus d'une génération. Le Collège royal des médecins et chirurgiens du Canada a récemment donné son aval concernant un programme de résidence en médecine de la douleur, et l'Université Western Ontario verra son premier diplômé canadien achever ce programme d'ici un an. Ce programme de résidence a été appuyé par la
Société canadienne des anesthésiologistes ainsi que la Société canadienne de la douleur et l'Association des départements d'anesthésie des universités canadiennes. La Dre Patricia Morley-Forster, une anesthésiologiste de l'Université Western, a pris la tête de ce processus. La génération future de spécialistes de la douleur proviendra de plusieurs spécialités, et il s'agit là d'un mécanisme qui pourrait régler le problème de l'utilisation inadaptée des opioïdes; c'est pourquoi notre profession doit soutenir cette initiative. Le Collège des médecins de famille du Canada met actuellement au point un Certificat de compétence additionnelle en prise en charge de la douleur. Cette initiative prendra forme au cours des années à venir. Il s'agit d'une occasion supplémentaire pour nos experts de faire valoir leur leadership.

Peu importe que vous croyiez ou non au concept de «drogue d'introduction » ou à une toxicomanie découlant d'une exposition limitée telle que celle survenant dans un contexte périopératoire, il ne fait aucun doute que l'un de nos objectifs premiers doit être d'encourager la pratique la plus adaptée, la plus sécuritaire et présentant le plus important succès dans la fourniture des soins périopératoires et de l'analgésie. Nous avons un rôle de leadership à remplir en médecine périopératoire et en médecine de la douleur. Par conséquent, nous devons nous engager de façon active face à nos collègues en chirurgie, en psychologie et en sciences de la réadaptation. Nous devons nous assurer que nous contribuons à la santé, et ne pas faire de tort à nos patients, et que nos soins ne se limitent pas à de brèves rencontres. Nous devons être conscients de et nous engager tout au long du cheminement du patient dans notre système.

Nous ne connaissons pas assez bien l'impact à long terme de l'utilisation d'opioïdes, et nous devons militer pour que des recherches pertinentes soient menées à terme dans ce domaine. Nous devons proposer des études, y prendre part, et suivre les patients via la création de registres qui nous permettront de mettre en place des stratégies de suivi afin de nous assurer que nos actes sont véritablement bénéfiques pour nos patients.

D'aucuns pourraient trouver l'article d'Alam et Juurlink rebutant, voire irritant en raison de sa source, de son contenu ou de son style. Mon conseil : «passez à autre chose », et concentrez-vous sur leur message. Leur article contient plusieurs messages clés auxquels nous, les anesthésiologistes, devons porter une attention particulière, ainsi que des initiatives nationales dans lesquelles nous devrions nous impliquer. Voilà, selon moi, le message clé de cet article. Ce manuscrit est un appel à l'acte-à participer à la diffusion des soins les meilleurs qui soient; à orienter les recherches sur lesquelles se fondent ces soins; et à éduquer les générations futures de professionnels de la santé en leur transmettant les 
compétences et les connaissances nécessaires à promouvoir la santé plutôt qu'à créer des problèmes de soins de santé iatrogènes.

Conflicts of interest None declared.

Disclosures Dr. Buckley has been an anesthesiologist and chronic pain physician since 1988, and he undoubtedly contributed to some of the widespread use of opioids described. He is also co-Chair (with Dr. David Mock, former Dean of Dentistry at University of Toronto) of the education implementation group of the Canadian Centre on Substance Abuse First Do No Harm (CCSA FDNH) strategy. He is director of the Michael G. DeGroote National Pain Centre which holds copyright for the "Canadian Guideline for Safe and Effective Use of Opioids for Chronic Non-Cancer Pain" and is responsible for its dissemination. The Guideline is currently under revision with funding from Health Canada.

\section{Conflit d'intérêt Aucun.}

Déclaration Dr Buckley est anesthésiologiste et médecin spécialiste de la douleur chronique depuis 1988 , et a indubitablement contribué à la propagation de l'utilisation des opioïdes d'ordonnance. Il est également co-président (avec Dr David Mock, ancien doyen de la faculté de médecine dentaire de l'Université de Toronto) du groupe de mise en oeuvre de l'éducation pour la Stratégie S'abstenir de faire du mal du Centre canadien de lutte contre les toxicomanies (CCLT). Il est directeur du Centre national de douleur Michael G. DeGroote, lequel détient les droits d'auteur et est responsable de la diffusion des Lignes directrices canadiennes sur l'utilisation sécuritaire et efficace des opiö̈des pour la douleur chronique non cancéreuse, un document actuellement en révision grâce au soutien financier de Santé Canada.

\section{References}

1. Alam A, Juurlink DN. The prescription opioid epidemic: an overview for anesthesiologists. Can J Anesth 2016; 63: this issue. DOI: 10.1007/s12630-015-0520-y.

2. National Opioid Use Guideline Group (NOUGG). Canadian Guideline for Safe and Effective Use of Opioids for Chronic Non-Cancer Pain-Version 4.5 April 30 2010. Available from URL: http://nationalpaincentre.mcmaster.ca/opioid/ (accessed October 2015).

3. Nuckols TK, Anderson L, Popescu I, et al. Opioid prescribing: a systematic review and critical appraisal of guidelines for chronic pain. Ann Intern Med 2014; 160: 38-47.

4. Canadian Centre on Substance Abuse. First Do No Harm: Responding to Canada's Prescription Drug Crisis. Annual Report 2013-2014. Available from URL: http://www.ccsa.ca/Resource\% 20Library/CCSA-Canada-Strategy-Prescription-Drug-MisuseAnnual-Report-2014-en.pdf (accessed October 2015). 